\title{
A linear algebra approach to OLAP
}

\author{
Hugo Daniel Macedo ${ }^{1}$ and José Nuno Oliveira ${ }^{2}$ \\ ${ }^{1}$ INRIA, Centre Paris-Rocquencourt, France \\ ${ }^{2}$ High Assurance Software Lab / INESC TEC \& Univ. Minho, Portugal
}

\begin{abstract}
Inspired by the relational algebra of data processing, this paper addresses the foundations of data analytical processing from a linear algebra perspective. The paper investigates, in particular, how aggregation operations such as cross tabulations and data cubes essential to quantitative analysis of data can be expressed solely in terms of matrix multiplication, transposition and the Khatri-Rao variant of the Kronecker product.

The approach offers a basis for deriving an algebraic theory of data consolidation, handling the quantitative as well as qualitative sides of data science in a natural, elegant and typed way. It also shows potential for parallel analytical processing (OLAP), as the parallelization theory of such matrix operations is well acknowledged.
\end{abstract}

Keywords: Software engineering ; Formal methods ; Data science

\section{Introduction}

In a recent article in the Harvard Business Review, Davenport and Patil DP12 declare data scientist as the sexiest job of the 21st century. Such high-ranking professionals should be trained to make discoveries in the world of big data, this showing how much companies are wrestling with information that comes in volumes never encountered before. The job calls for a lot of creativity mixed with solid foundations in maths, statistics, probability, and computer science.

Leaving aside the enormous challenges posed by big unstructured data, a data scientist is expected to live on data science, whatever this is. Concerning structured data, we see data science as a two-fold body of knowledge, made of qualitative as well as quantitative ingredients. The qualitative side is provided by the solid theory of databases Mai83 which, formalized in logic and (relational) set theory, has led to standard querying languages over relational data such as SQL. As for the quantitative side, we see similar efforts in the formalization of data analytic techniques — put forward under the umbrella of the OLAP ${ }^{1}$ acronym — but such efforts seem less successful in setting up a thorough semantic basis for understanding and optimizing analytical processing.

It is true that formal definitions for concepts such as multi-dimension database [GL97, data aggregation

Correspondence and offprint requests to: Hugo Daniel Macedo, INRIA, 23 avenue d'Italie, CS 81321, 75214 Paris Cedex 13, France. e-mail: hugo.dos_santos_macedo@inria.fr

1 OLAP stands for On-line Analytical Processing [DT99, PJ01]. 
and data cube DT99 have been given (among others), including an algebra of cube operators DT99. Little is written, however, concerning algebraic properties of such operators. And those which are given either address the qualitative side again (the dimension algebra [JLN00 rather than the measure one) or are stated without proof (e.g. the two equalities in [GCB+97] concerning roll-up, group-by and cube).

These shortcomings are easy to understand: while relation algebra "à la Codd" [Cod70] and naive set theory work well for qualitative data science (focus on attribute and dimension structures), they are rather clumsy in handling the quantitative side (focus on measure structures and their operations). In this paper we propose to solve this problem by suggesting linear algebra (LA) as an alternative suiting both sides: the qualitative one - by regarding it as a typed theory — and the quantitative one — by internalizing all details of data consolidation and aggregation under the operations of matrix composition (namely multiplication) and converse (transposition).

This approach builds upon previous work on typed linear algebra and its applications in computer science, which include areas as diverse as data vectorization MO13, probabilistic program calculation Oli12, weighted automata Oli13, component-oriented design MO11b, Oli14b etc. Details and further examples can be found in a technical report MO11a which also elaborates on the potential of the approach for OLAP parallelization.

Contribution. The ideas presented in this paper derive from the authors' work on typing linear algebra MO10, Mac12, MO13 which eventually drove them into the proposed synergy between linear algebra and OLAP. Such a synergy is, to the best of their knowledge, novel in the field. Rather than relying on standard OLAP state of the art developments, a cross-field perspective is put forward that may open new ways of looking at this body of knowledge.

Overview of the paper. The remainder of this paper is structured as follows. Sections 2 and 3 explain the shift from relational to linear algebra, imposed by the shift from qualitative to quantitative processing. Section 4 gives a brief overview of typed linear algebra. Section 5 expresses cross tabulations solely in terms of linear algebra matrix operations. Section 6 treats cross tabulation and "rolling up" along functional dependencies, introducing dimension hierarchies into the game. Section 7 proves that the construction of cross tabulations is incremental. Section 8 goes higher-dimensional into the LA construction of OLAP cubes. Finally, section 9 reviews related work and section 10 draws conclusions and gives a prospect of future work. Some technical details and proofs are deferred to the two appendices.

\section{From relations to matrices}

On-line analytical processing [DT99, PJ01, JPT10, aims at summarizing huge amounts of information in the form of histograms, sub-totals, cross tabulations (namely pivot tables), roll-up/drill-down transformations and data cubes, whereby new trends and relationships hidden in raw data can be found. The need for this technology concerns not only large companies generating huge amounts of data every day (the "big data" trend) but also the laptop spreadsheet user who wants to make sense of the data stored in a particular workbook.

Since Codd's pioneering work on the foundations of the relational data model [Cod70], relation algebra has been adopted as the standard basis for formalizing data processing. Given the proximity between relation and matrix algebra SSch11, DGM14 the question arises: how much gain can one expect from translating results from one side to the other? This paper will show how a particular construction in relation algebra - that of a binary relational projection, defined in Oli09, Oli11 to calculate with functional dependencies in databases - translates matrix-wise into cross tabulations (namely pivot tables) which are central to data analytical processing.

On the relational side, a binary relational projection is always of the form

$$
\pi_{f, g} R=\{(f b, g a) \mid(b, a) \in R\}
$$

where $R$ is the binary relation being projected and $f$ and $g$ are observation functions, usually associated to attributes. Although less common in the database literature, the alternative definition

$$
\pi_{f, g} R=f \cdot R \cdot g^{\circ}
$$




\begin{tabular}{|c|l|r|l|r|}
\hline Line & Model & Year & Color & Sales \\
\hline 1 & Chevy & 1990 & Red & 5 \\
\hline 2 & Chevy & 1990 & Blue & 87 \\
\hline 3 & Ford & 1990 & Green & 64 \\
\hline 4 & Ford & 1990 & Blue & 99 \\
\hline 5 & Ford & 1991 & Red & 8 \\
\hline 6 & Ford & 1991 & Blue & 7 \\
\hline
\end{tabular}

Fig. 1. Collection of raw data (adapted from GC97]).

is simpler and easier to reason about, where the dot $(\cdot)$ between the symbols denotes relational composition and ( $)^{\circ}$ expresses the converse operation: pair $(b, a)$ belongs to relation $R^{\circ}$ iff pair $(a, b)$ belongs to $R{ }^{2}$

Projection pattern (1) turns up often in relation algebra BdM97. When expressing data dependencies, such projections take the form

$$
f_{A} \cdot \llbracket T \rrbracket \cdot f_{B}^{\circ}
$$

where $T$ is a database file, or table (a set of data records, or tuples), $A$ and $B$ are attributes of the schema of $T, f_{A}$ (resp. $\left.f_{B}\right)$ is the function which captures the semantics of attribute $A$ (resp. $\left.B\right) \rrbracket^{3}$ and $\llbracket T \rrbracket$ represents set $T$ in the form of a diagonal relation:

$$
\llbracket T \rrbracket=\{(t, t) \mid t \in T\}
$$

This somewhat redundant construction proves essential to the reasoning, as shown in Oli11, Oli14a. Expressed in set-theoretical notation, projection $[2]$ is set-comprehension $\{(t[A], t[B]) \mid t \in T\}$ where $t[A]$ (resp. $t[B])$ denotes the value of attribute $A$ (resp. $B$ ) in tuple $t$.

Note how simple $\sqrt{2}$ is in its relying only on very basic combinators of relation algebra, namely composition and converse, which generalize to matrix multiplication and transposition, respectively. Under this generalization, we will show below that cross tabulations can be expressed by a formula similar to (2),

$$
t_{A} \cdot \llbracket T \rrbracket_{M} \cdot t_{B}^{\circ}
$$

where $M$ is a measure attribute and attributes $A$ and $B$ are the dimensions chosen for each particular cross tabulation. Notation $t_{A}$ (resp. $t_{B}$ ) expresses the membership matrix of the column addressed by dimension $A$ (resp. $B$ ) whose construction will be explained later. Also explained later, $\llbracket T \rrbracket_{M}$ denotes the diagonal matrix capturing column $M$ of $T 4^{4}$

The construction of matrices $t_{A}, t_{B}$ and $\llbracket T \rrbracket_{M}$ will be first illustrated with examples. Cross tabulations will be pictured as displayed by Microsoft Excel.

\section{Cross-tabulations}

In data processing, a cross tabulation (or pivot table) provides a particular summary or view of data extracted from a raw data source. As example of raw data consider the table displayed in Figure 1 where each row records the number of vehicles of a given model and color sold per year.

In general, the raw data out of which cross tabulations are calculated is not normalized and is collected into a central database, termed a data warehouse or decision support database. Different summaries answer different questions such as, for instance, how many vehicles were sold per color and model? For this particular question, the attributes Color and Model are selected as dimensions of interest, Sales is regarded as measure attribute and the corresponding cross tabulation is depicted in Figure 2, as generated via the pivot table menu in Excel.

\footnotetext{
2 Recall from discrete maths that, given two relations $R$ and $S$, pair $(c, a)$ will be in the composition $R \cdot S$ iff there is some $b$ such that $(c, b)$ is in $R$ and $(b, a)$ is in $S$. Thus, $(y, x) \in f \cdot R \cdot g \circ$ in 11 means that $y=f b$ and $x=g a$ for some $(b, a) \in R$, that is, $(y, x)=(f b, g a)$. Altogether, $f \cdot R \cdot g^{\circ}=\bigcup_{(b, a) \in R}\{(f b, g a)\}$ which reduces to the given set comprehension.

3 That is, given a tuple $t \in T, f_{A}(t)$ yields the value of attribute $A$ in $t$, usually denoted by $t[A]$ (similarly for attribute $B$ ).

4 The shift from the binary relations of $\sqrt{2}$ to the matrices in 3 will be detailed in the sequel. Although relations can be represented by Boolean matrices containing only 0 s and 1 s (more about this in appendix $\mathrm{A}$, matrix $\llbracket T \rrbracket_{M}$ will be a numeric matrix in general holding real-life quantities and measures.
} 


\begin{tabular}{|l|r|r|r|}
\hline Sum of Sales & Model & & \\
\hline Color & Chevy & Ford & Grand Total \\
\hline Blue & 87 & 106 & 193 \\
Green & & 64 & 64 \\
Red & 5 & 8 & 13 \\
\hline Grand Total & 92 & 178 & 270 \\
\hline
\end{tabular}

Fig. 2. Pivot table as extracted by Excel from the data in Figure 1.

Large scale cross tabulation generation is an essential part of quantitative data analysis. As already mentioned, OLAP refers to the set of techniques performing such analysis over information stored in data warehouses, whose complexity is well-known [PKL02. Quoting [DT99]: The complexity of queries required to support OLAP applications makes it difficult to implement using standard relational database technology. Feeling the lack of a standard conceptual model for OLAP, the same authors [DT99. propose one based on first order logic. Reference [VS99] provides a review of other efforts in defining logical models for OLAP.

Rather than trying to extend existing logic models towards accommodating OLAP semantics, the approach put forward in this paper changes strategy and calls for a synergy with the field of linear algebra. The key resides in expressing analytic operations in the form of matrix algebra expressions. In the particular case of reporting multi-dimensional analyses of data, one should be able to build three matrices as hinted by formula (3): two associated to the dimensions (attributes) $A$ and $B$ being analysed and a third one recording which measure or metric data are to be considered for consolidation.

This encoding of data into LA is quite smooth if matrix operations are typed in the way presented in e.g. MO13. For self-containedness we give a very brief overview of such typed $L A$ notation below.

\section{Typed linear algebra}

Matrices as arrows. A matrix $M$ with $n$ rows and $m$ columns is a function which tells the value $r M c$ which occupies the cell addressed by row $r$ and column $c$, for $1 \leqslant r \leqslant n, 1 \leqslant c \leqslant m$. Note that we prefer infix notation $r M c$ to e.g. $M_{r c}$ or even $M(r, c)$ for reasons to be explained later.

Following the arrow notation of [MO13] and writing $n<M-m$ to denote that matrix $M$ is of type $n \leftarrow m$ ( $m$ columns, $n$ rows), matrix multiplication can be expressed by arrow composition:

$$
n \underbrace{M}_{C=M \cdot N} m \ll N
$$

Point-wise, this operation is defined by ${ }^{5}$

$$
y(M \cdot N) x=\langle\Sigma z:: y M z \times z N x\rangle
$$

For every $n$ there is a matrix of type $n \leftarrow n$ which is the unit of composition.

This is nothing but the identity matrix of size $n$, denoted by $n \ll \stackrel{i d_{n}}{\leftarrow}$ or $n<1$ indistinguishably. Therefore (diagram aside):

$$
i d_{m} \cdot M=M=M \cdot i d_{n}
$$

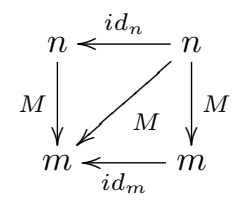

Subscripts $m$ and $n$ can be omitted wherever the underlying diagrams are well-defined and can be inferred from the context.

\footnotetext{
5 This and other pointwise definitions and rules to come are expressed in the style of the Eindhoven quantifier calculus, see e.g. BM06. Matrix multiplication is so-called because it can be regarded as an extension of numeric multiplication to matrices. Phrase matrix composition emphasises the underlying categorial basis [MO13] of this operation, which is less widely acknowledged. As types are central to the approach proposed in this paper, we will write composition instead of multiplication unless quoting work which explicitly uses the latter terminology.
} 
Vectors as arrows. Vectors are special cases of matrices in which one of the dimensions is 1 , for instance

$$
v=\left[\begin{array}{c}
v_{1} \\
\vdots \\
v_{m}
\end{array}\right] \quad \text { and } \quad w=\left[\begin{array}{lll}
w_{1} & \ldots & w_{n}
\end{array}\right]
$$

Column vector $v$ is of type $m \leftarrow 1$ ( $m$ rows, one column) and row vector $w$ is of type $1 \leftarrow n$ (one row, $n$ columns). Our convention is that lowercase letters (e.g. $v, w$ ) denote vectors and uppercase letters (e.g. $M$, $N$ ) denote arbitrary matrices.

Converse of a matrix. One of the kernel operations of linear algebra is transposition, whereby a given matrix changes shape by turning its rows into columns and vice-versa. Given matrix $n<^{M} m$, notation $m M^{M^{\circ}} n$ denotes its transpose, or converse. The following laws hold: $\left(M^{\circ}\right)^{\circ}=M$ (idempotence) and $(M \cdot N)^{\circ}=N^{\circ} \cdot M^{\circ}$ (contravariance).

Block notation. Matrices can be built of other matrices using block notation. Two basic binary combinators are identified in MO13 for building matrices out of other matrices, say $M$ and $N$, regarded as blocks, either stacking these vertically, $\left[\begin{array}{l}M \\ N\end{array}\right]$, or horizontally, $[M \mid N]$. Dimensions should agree, as shown in the diagram aside, taken from MO13, where $m, n, p$ and $t$ are types. Special matrices $i_{1}, i_{2}, \pi_{1}$ and $\pi_{2}$ are fragments of the identity matrix and play an important role in explaining the semantics of the two combinators. This, however, can be skipped for the purposes of the current paper 6 , sufficing to know a number of laws which emerge from the underlying mathematics, namely converse-duality

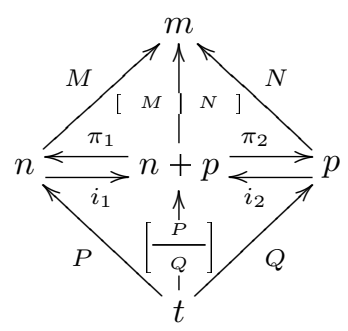

$$
[M \mid N]^{\circ}=\left[\frac{M^{\circ}}{N^{\circ}}\right]
$$

divide-and-conquer

$$
[M \mid N] \cdot\left[\frac{P}{Q}\right]=M \cdot P+N \cdot Q
$$

which captures the essence of (parallelizable) matrix multiplication, two fusion laws

$$
\begin{aligned}
P \cdot[M \mid N] & =[P \cdot M \mid P \cdot N] \\
{\left[\frac{M}{N}\right] \cdot P } & =\left[\frac{M \cdot P}{N \cdot P}\right]
\end{aligned}
$$

and the abide law 7

$$
\left[\begin{array}{c|c}
{[M \mid N]} \\
\hline[P \mid Q]
\end{array}\right]=\left[\left[\frac{M}{P}\right] \mid\left[\frac{N}{Q Q}\right]\right]=\left[\begin{array}{l|l}
M & N \\
\hline P & Q
\end{array}\right]
$$

which establishes the equivalence between row-major and column-major construction of matrices by blocks. (Thus the four-block notation on the right.)

\footnotetext{
${ }_{6}$ The rich algebra of matrix block-operations arises essentially from the fact that vertical and horizontal block aggregation form a biproduct. The interested reader is referred to MO13 for details.

7 Neologism "abide" (= "above and beside") was introduced by Richard Bird [Bir89] as a generic name for algebraic laws in which two binary operators written in infix form change place between "above" and "beside", e.g.
}

$$
\frac{a}{b} \times \frac{c}{d}=\frac{a \times c}{b \times d}
$$

in fraction calculus. 
Direct sum and Kronecker product. Given two matrices $M$ and $N$, the direct sum of $M$ and $N$ is defined as follows, using block notation:

$$
M \oplus N=\left[\begin{array}{c|c}
M & 0 \\
\hline 0 & N
\end{array}\right]
$$

Mind the type $k+j \stackrel{M \oplus N}{\longleftarrow} n+m$ for $M$ and $N$ of types $k \leftarrow n$ and $j \leftarrow m$, respectively. Direct sum is a standard linear algebra operator enjoying many useful properties MO13. The following equation, termed the absorption law, specifies how block operator [ | ] absorbs direct sum $\oplus$, for suitably typed matrices $M, N, P$ and $Q$ :

$$
[M \mid N] \cdot(P \oplus Q)=[M \cdot P \mid N \cdot Q]
$$

Given the same two matrices $k \ll^{M} n$ and $j<^{N} m$, another standard construction in linear algebra is the so-called Kronecker product $k \times j \stackrel{M \otimes N}{\longleftarrow} n \times m$. This operator can be defined by block-wise decomposition,

$$
\begin{aligned}
{[M \mid N] \otimes P } & =[M \otimes P \mid N \otimes P] \\
{\left[\frac{M}{N}\right] \otimes P } & =\left[\frac{M \otimes P}{N \otimes P}\right] \\
x \otimes N & =x N
\end{aligned}
$$

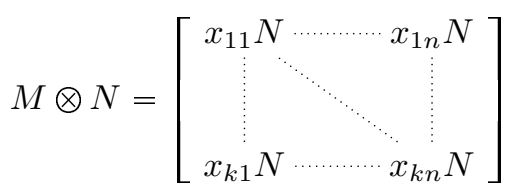

where $x$ is a scalar (1-to-1 matrix) and $x N$ denotes scalar multiplication. The picture above describes the outcome of the operation.

Khatri-Rao matrix product. Given matrices $n<^{M} m$ and $p<^{N} m$, the so-called Khatri-Rao [RR98 matrix product of $M$ and $N$, denoted $n \times p \stackrel{M \nabla N}{\longleftarrow} m$ is a column-wise version of the Kronecker product operator given above,

$$
\begin{aligned}
u \nabla v & =u \otimes v \\
{\left[M_{1} \mid M_{2}\right] \nabla\left[N_{1} \mid N_{2}\right] } & =\left[M_{1} \nabla N_{1} \mid M_{2} \nabla N_{2}\right]
\end{aligned}
$$

where $u, v$ are column-vectors and $M_{i}, N_{i}$ are suitably typed matrices 8 As an example of operation relying on this product consider row vector

$$
s=\left[\begin{array}{llllll}
5 & 87 & 64 & 99 & 8 & 7
\end{array}\right]
$$

of type $1 \longleftarrow^{s} 6$, capturing the transposition of the Sales column of Figure 1 . The Khatri-Rao product $s \nabla i d$ yields the corresponding diagonal matrix:

$$
6 \stackrel{\text { svid }}{<} 6=\left[\begin{array}{cccccc}
5 & 0 & 0 & 0 & 0 & 0 \\
0 & 87 & 0 & 0 & 0 & 0 \\
0 & 0 & 64 & 0 & 0 & 0 \\
0 & 0 & 0 & 99 & 0 & 0 \\
0 & 0 & 0 & 0 & 8 & 0 \\
0 & 0 & 0 & 0 & 0 & 7
\end{array}\right]
$$

This conversion is essential to the LA encoding of cross tabulations, as shown in the sequel.

One can reduce over a matrix defined by rows on the right-hand side of a Khatri-Rao product whose left-hand side is a row vector:

$$
v \nabla\left[\frac{M}{N}\right]=\left[\frac{v \nabla M}{v \nabla N}\right] .
$$

8 As shown in Mac12], this product generalizes to arbitrary matrices the tupling operator known as split in the functional setting [BdM97] or as fork in the relational one [Fri02, Sch11]. 
Should the shape of the matrix on the right hand side be a direct sum, the equation can be rewritten into:

$$
[v \mid w] \nabla(M \oplus N)=(v \nabla M) \oplus(w \nabla N)
$$

This follows from 15 and $(13)$.

Type generalization. Matrix types (the end points of arrows) can be generalized from traditional numeric dimensions to arbitrary denumerable types thanks to addition and multiplication of matrix elements being commutative and associative. This ensures unambiguous definition of matrix composition because the summation inside the inner product of two vectors (5) can be calculated in any order. Typewise, our convention is that lowercase letters (e.g. $n, m$ ) denote the traditional dimension types (natural numbers), letting uppercase letters (e.g. $A, B$ ) denote other types and taking disjoint union $A+B$ for $m+n$, Cartesian product $A \times B$ for $m n$, unit type 1 for number 1 , the empty set $\varnothing$ for 0 and so on. Conversely, dimension $n$ corresponds to the initial segment $\{1,2, \ldots, n\}$ of the natural numbers up to $n$.

There is another "type" associated with matrices, namely the type of the elements (cells). The default view in linear algebra is to regard them as complex or real numbers, or (more generically) as inhabitants of an algebraic field. The minimal structure for composition (5) to work is that of a semiring, e.g. the natural numbers $\left(\mathbb{N}_{0}\right)$ under addition and multiplication. Matrices whose cells are $\mathbb{N}_{0}$-valued are referred to as counting matrices and addressed in appendix A. They include so-called Boolean matrices, whose cells are either 0 or 19

\section{Cross tabulations in LA}

Recall that the core of cross tabulation generation is formula (3), which is the matrix counterpart to relational projection (2). This section explains this construct starting by showing how the move from relations to matrices is obtained by encoding functions as matrices.

Building projection functions. Let $A$ be an attribute of raw-data table $T$ and let $n$ be the number of records in $T$ (namely rows, or lines in a spreadsheet). We write $T(A)$ to denote the column of $T$ identified by attribute $A, T(A, y)$ to denote the element occupying the $y$-th position (row) in such a column, and $|A|$ to denote the range of values which can be found in $T(A)$. Column $T(A)$ can be regarded as a function which tells, for each row number $1 \leqslant r \leqslant n$, which value in $|A|$ can be found in row $r$ of such a column. Such a function can be encoded as an elementary matrix $t_{A}$ of type $|A| \leftarrow n$, defined as follows:

$$
a t_{A} r= \begin{cases}1 & \text { if } T(A, r)=a \\ 0 & \text { otherwise }\end{cases}
$$

These projections can be identified with the bitmaps of WOS06, regarded as matrices. In our running example (Figures 1 and 2 $n=6$ and we want to build these matrices for attributes Model and Color. The projection $\mid$ Model $\mid \stackrel{t_{\text {Model }}}{\longleftarrow} n$ associated to dimension Model is matrix

\begin{tabular}{ccccccc} 
& 1 & 2 & 3 & 4 & 5 & 6 \\
\hline Chevy & 1 & 1 & 0 & 0 & 0 & 0 \\
Ford & 0 & 0 & 1 & 1 & 1 & 1
\end{tabular}

and projection $\mid$ Color $\mid \stackrel{t_{\text {Color }}}{\longleftarrow} n$ associated to dimension Color is matrix

\begin{tabular}{ccccccc} 
& 1 & 2 & 3 & 4 & 5 & 6 \\
\hline Blue & 0 & 1 & 0 & 1 & 0 & 1 \\
Green & 0 & 0 & 1 & 0 & 0 & 0 \\
Red & 1 & 0 & 0 & 0 & 1 & 0
\end{tabular}

\footnotetext{
9 Boolean operations can be implemented in $\{0,1\} \subseteq \mathbb{N}_{0}$ by defining $a \wedge b=a b, a \vee b=a+b-a b$ and $\neg a=1-a$, which are all closed in $\{0,1\}$. This is not, however, required in the sequel.
} 
Note that, typewise, the composition of matrices $t_{C o l o r}$ and $t_{\text {Model }}^{\circ}$ makes sense, leading to matrix

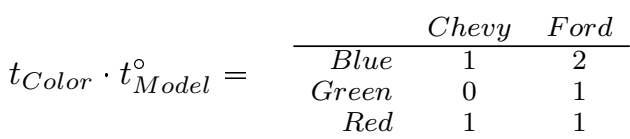

of type $\mid$ Color $|\leftarrow|$ Model $\mid$, which essentially counts the number of sale records per color and model. In general, given attribute values $a \in|A|$ and $b \in|B|$, the cell in $t_{A} \cdot t_{B}^{\circ}$ addressed by $a$ and $b$ counts the number of rows of the source dataset $T$ in which both $a$ and $b$ occur in the $A$ and $B$ columns, respectively:

$$
a\left(t_{A} \cdot t_{B}^{\circ}\right) b=\langle\Sigma n: T(A, n)=a \wedge T(B, n)=b: 1\rangle
$$

The derivation of 21 will be given shortly ${ }^{10}$

The diagonal construction. In order to sum up the number of vehicles sold rather than just counting sale records we need to identify a measure attribute, that is, a numeric attribute of $T$ to be used for consolidation. In the case of Figure 1 only Sales applies. Because such numeric data have to become available for both projection matrices of (3), to the left and to the right, the chosen column is converted into a diagonal matrix as already shown in (14).

Notation $\llbracket T \rrbracket_{M}$ will be used to denote the diagonal matrix representation of measure attribute $M$ in $T$. Index-wise, this corresponds to the following definition:

$$
j \llbracket T \rrbracket_{M} i= \begin{cases}T(M, j) & \text { if } i=j \\ 0 & \text { otherwise }\end{cases}
$$

Definition 72 in appendix B gives a pointfree alternative to 22 which is better suited for calculational purposes.

LA script for cross tabulation. We are in position to run formula (3) for $T$ as in Figure 1 , $A=C o l o r$ and $B=$ Model. The evaluation of $t_{\text {Color }} \cdot \llbracket T \rrbracket_{\text {Sales }} \cdot t_{\text {Model }}^{\circ}$ yields another matrix of type $\mid$ Color $|\leftarrow| M o d e l \mid$

\begin{tabular}{rcc} 
& Chevy & Ford \\
\hline Blue & 87 & 106 \\
Green & 0 & 64 \\
Red & 5 & 8
\end{tabular}

which we will denote by $\operatorname{ctab}_{\text {Color } \leftarrow \text { Model }}^{\text {Sales }}(T)$ relying on the definition

$$
\begin{aligned}
& \operatorname{ctab}_{A \leftarrow B}^{M}(T):|A| \leftarrow|B| \\
& \operatorname{ctab}_{A \leftarrow B}^{M}(T)=t_{A} \cdot \llbracket T \rrbracket_{M} \cdot t_{B}^{\circ}
\end{aligned}
$$

— recall $(3)$ — whose pointwise meaning is

$$
a\left(\operatorname{ctab}_{A \leftarrow B}^{M}(T)\right) b=\left\langle\sum n: T(A, n)=a \wedge T(B, n)=b: T(M, n)\right\rangle
$$

as will be shown briefly. In words: we sum all cells $T(M, n)$ with $n$ ranging over all rows such that $T(A, n)$ and $T(B, n)$ respectively hold the attribute values $a$ and $b$ being consolidated (ie. related). The derivation of 25 relies on some rules for pointwise matrix manipulation given in appendix A. Note the style of the equational proof where each step is labeled with references to the laws applied, written inside the curly

\footnotetext{
10 This situation (counting), which is what Excel outputs wherever the measure attribute chosen in pivot table calculation is
} not numeric, corresponds to formula (3) wherever the middle matrix is the identity. 
braces that follow the equality symbol (=):

$$
\begin{aligned}
& a\left(\operatorname{ctab}_{A \leftarrow B}^{M}(T)\right) b \\
& =\quad\{\text { definition } 24\} \\
& a\left(t_{A} \cdot \llbracket T \rrbracket_{M} \cdot t_{B}^{\circ}\right) b \\
& =\quad\left\{\text { matrix composition } 55 \text { twice; converse of } t_{B}\right\} \\
& \left\langle\Sigma n::\left(a t_{A} n\right) \times\left\langle\Sigma m::\left(n \llbracket T_{M} \rrbracket m\right) \times\left(b t_{B} m\right)\right\rangle\right\rangle \\
& =\left\{\llbracket T_{M} \rrbracket \text { is diagonal } 22\right\} \\
& \left\langle\Sigma n::\left(a t_{A} n\right) \times\left\langle\Sigma m: m=n:\left(n \llbracket T_{M} \rrbracket m\right) \times\left(b t_{B} m\right)\right\rangle\right\rangle \\
& =\quad\{\text { one point rule (quantifying over } m=n \text { ) }\} \\
& \left\langle\Sigma n::\left(a t_{A} n\right) \times\left(n \llbracket T_{M} \rrbracket n\right) \times\left(b t_{B} n\right)\right\rangle \\
& =\quad\left\{\text { "trading" over Boolean cells } a t_{A} n \text { and } b t_{B} n \text { (see appendix A }\right\} \\
& \left\langle\Sigma n: a t_{A} n \wedge b t_{B} n: n \llbracket T_{M} \rrbracket n\right\rangle \\
& =\quad\left\{\text { pointwise meaning of projections } t_{A}, t_{B} \llbracket 17 \text { and diagonal } \llbracket T_{M} \rrbracket 22\right\} \\
& \langle\Sigma n: T(A, n)=a \wedge T(B, n)=b: T(M, n)\rangle
\end{aligned}
$$

Clearly, 21] is a corollary of 225 since, for $\llbracket T_{M} \rrbracket=i d, n \llbracket T_{M} \rrbracket n=1$ :

$$
a\left(t_{A} \cdot t_{B}^{\circ}\right) b=a\left(t_{A} \cdot i d \cdot t_{B}^{\circ}\right) b=\langle\Sigma n: T(A, n)=a \wedge T(B, n)=b: 1\rangle
$$

Grand totals. If compared to Figure 2 , cross tabulation 23 misses the two row and column grand totals. These are easily obtained via "bang" matrices. Let us explain what these are and our choice of terminology. In functional programing, the popular "bang" function, which is of type $1 \leftarrow A$ (parametric on $A, \forall A$ ) and usually denoted by symbol "!", is a polymorphic constant function yielding the unique value which inhabits the singleton type 1 . The encoding of this function in LA format will be the row vector $1 \stackrel{!_{A}}{\leftarrow} A$ wholly filled up with 1 s. For instance, $!_{\mid M o d e l} \mid$ will be the vector with $\mid$ Model $\mid$-many positions all holding number 111

Clearly, the composition of row vector $1 \longleftarrow^{!} A$ with any column vector of type $A \longleftarrow^{v} 1$ computes a scalar: the sum of all cells in $v$. Thus one can define a generic totalizer operator,

$$
\text { tot } X=\left[\frac{i d}{!}\right] \cdot X \cdot\left[\frac{i d}{!}\right]^{\circ}
$$

which equips $X$ with three other blocks

$$
\left[\begin{array}{c|c}
X & X \cdot !^{\circ} \\
\hline ! \cdot X & ! \cdot X \cdot !^{\circ}
\end{array}\right]
$$

two sum (row and column) vectors and the grand total scalar $! \cdot X \cdot !^{\circ}$ 12 By adding totals to ctab 24 we

11 For the purposes in this paper, type 1 can be regarded as the singleton set $\{$ ALL $\}$. This corresponds to the Grand Total in Figure 2 and is consistent with the way ALL is used in e.g. [GCB ${ }^{+97}$, as explained later in section 8

12 The transformation of 26 into 27 follows immediately from the matrix laws of section 4 
define

$$
\begin{aligned}
& \operatorname{tctab}_{A \leftarrow B}^{M}(T):|A|+1 \leftarrow|B|+1 \\
& \operatorname{tctab}_{A \leftarrow B}^{M}(T)=\operatorname{tot}\left(\operatorname{ctab}_{A \leftarrow B}^{M}(T)\right)=\left[\frac{t_{A}}{!}\right] \cdot \llbracket T \rrbracket_{M} \cdot\left[\frac{t_{B}}{!}\right]^{\circ}
\end{aligned}
$$

which computes the standard cross-tabulation of raw data table $T$ with respect to dimensions $A, B$ and measure $M$. Note how types (dimensions) are added with 1, the singleton type containing the distinguished element ALL labelling grand totals. In our running example, this corresponds to enriching (23) with the extra row and column corresponding to the bang vectors of (26), both labeled with ALL:

\begin{tabular}{rccc} 
& Chevy & Ford & ALL \\
\hline Blue & 87 & 106 & 193 \\
Green & 0 & 64 & 64 \\
Red & 5 & 8 & 13 \\
ALL & 92 & 178 & 270
\end{tabular}

Such is the outcome of evaluating $t c t a b_{\text {Color } \leftarrow \text { Model }}^{\text {Sales }}(T)$, which finally achieves the effect of Figure 2 involving LA operations only.

As illustration of how these LA-based operations can be encoded in commercial languages dealing with matrices, such as e.g. MATLAB ${ }^{13}$, listing 1 provides MATLAB code for the generation of the bang vector of size $r$, the tot operator (26) and the calculation of cross tabulations $(2428)$.

Finally, among several properties of bang vectors we single out

$$
\begin{aligned}
& {[! \mid !]=!} \\
& ! \nabla A=A=A \nabla !
\end{aligned}
$$

where (31) identifies ! as the unit of Khatri-Rao product. Since this is associative too, one can rely on its finitary extension to a sequence of $n$ matrices $A_{i}$ (all sharing the same input type, for $1 \leqslant i \leqslant n$ ) by writing $\nabla_{i=1}^{n} A_{i}$ or even

$$
\underset{i \leftarrow s}{\nabla} A_{i}
$$

where $s$ is a finite sequence of indices ${ }^{14}$ This extension will be useful in the generation of data cubes to be given in section 8 Prior to this, we address below another operation central to OLAP: roll-up.

\section{6. "Rolling up" on functional dependencies}

Rolling up means replacing a dimension by another which is more general in some sense (e.g. grouping, classification, containment). The latter is therefore "higher" in a dimension hierarchy which somehow acts as a classification or taxonomy of data records.

A simple way of seeing roll-up at work is the acknowledgement of functional dependencies (FDs) in data Mai83. Let us, for instance, augment the raw data of our running example with two new columns recording the month and season of each sale, as displayed in Figure 3 Look, for instance, at the column labelled Season telling in which season (Spring, Summer, Autumn or Winter) the particular sales took place. Clearly, FD Season $\leftarrow$ Month holds, as no sales are recorded in the same month and in different seasons. This possibly happens because the Season and Month columns result from a join of the original table with some other table recording that Season is higher than Month in the temporal dimension hierarchy ${ }^{15}$

\footnotetext{
13 MATLAB TM is a trademark of The MathWorks $®$.

14 Thus $\nabla_{i \leftarrow \square]} A_{i}=!$ and $\nabla_{i \leftarrow(k: s)}=A_{k \nabla}\left(\nabla_{i \leftarrow s} A_{i}\right)$, where $[$ denotes the empty sequence and $(k: s)$ denotes the appending of head $k$ to sequence $s$.

15 The fact that $T$ is not normalized in general reflects the preparation process of merging into the same data warehouse different tables of a (normalized) database.
} 


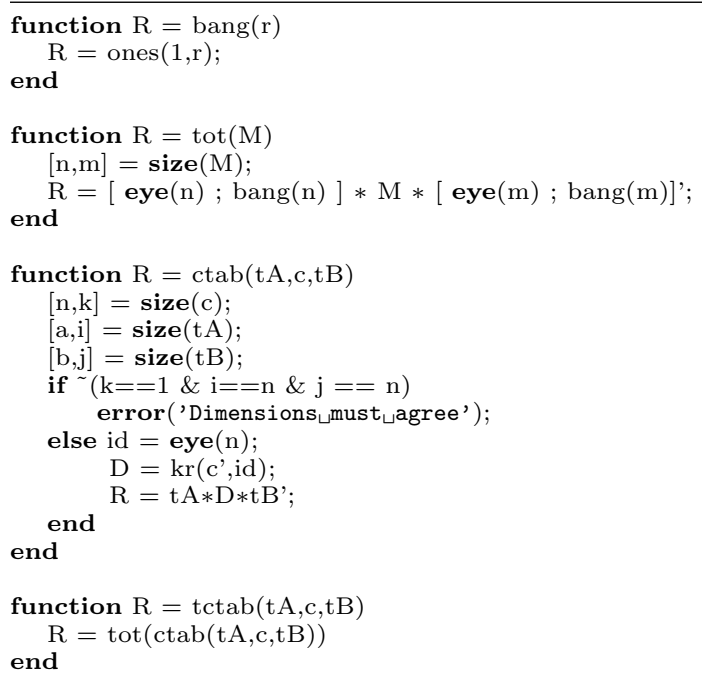

Listing 1: MATLAB encoding of bang (!), tot and of cross table calculation (ctab and tctab), where the measure column is parameter $c$ (a vector). This is converted to a diagonal as in (14) via the Khatri-Rao auxiliary operator $k r$ taken from the Tensorlab library [SBL14.

\begin{tabular}{|l|r|l|r|l|l|}
\hline Model & Year & Color & Sales & Month & Season \\
\hline Chevy & 1990 & Red & 5 & March & Spring \\
\hline Chevy & 1990 & Blue & 87 & April & Spring \\
\hline Ford & 1990 & Green & 64 & August & Summer \\
\hline Ford & 1990 & Blue & 99 & October & Autumn \\
\hline Ford & 1991 & Red & 8 & January & Winter \\
\hline Ford & 1991 & Blue & 7 & January & Winter \\
\hline
\end{tabular}

Fig. 3. Augmented collection of raw data.

Roll-up matrices. In general, a functional dependency $B \leftarrow A$ will hold in a table $T$ iff no pair of rows can be found in $T$ in which the values of attribute $A$ are the same and those of attribute $B$ differ (" $B$ is determined by $\left.A^{\prime \prime}\right)$ :

$$
\langle\forall n, m: T(A, n)=T(A, m): T(B, n)=T(B, m)\rangle
$$

In the style of Oli14a, we will write $B<^{T} A$ to mean 33 , abbreviated to $B \leftarrow A$ wherever $T$ is implicit. As is shown in appendix A, (33) can be expressed solely in terms of projection matrices:

$$
B \ll{ }^{T} A \Leftrightarrow t_{A}^{\circ} \cdot t_{A} \leqslant t_{B}^{\circ} \cdot t_{B}
$$

Whenever $B \stackrel{T}{\leftarrow} A$ holds, $B$ acts as a classifier for $A$, meaning that every cross tabulation involving $A$ can be rolled-up into another (less detailed) one involving $B$ instead. In general, we define the roll-up matrix $|B| \stackrel{t_{B \leftarrow A}}{\longleftarrow}|A|$ associated to FD $B \leftarrow A$ by

$$
t_{B \leftarrow A}=\left\lfloor t_{B} \cdot t_{A}^{\circ}\right\rfloor
$$

where $\lfloor M\rfloor$ denotes the support of a given matrix $M(59)$ : the matrix of the same type whose non-zero cells are mapped to 1 .

For instance, let us compute $t_{\text {Season }} \cdot t_{\text {Month }}^{\circ}$ (aside). This is a matrix of natural numbers counting the number of records in which a particular relationship holds, for instance January versus Winter, which turns up twice. Quantities

\begin{tabular}{rccccc} 
& January & March & April & August & October \\
\hline Spring & 0 & 1 & 1 & 0 & 0 \\
Summer & 0 & 0 & 0 & 1 & 0 \\
Autumn & 0 & 0 & 0 & 0 & 1 \\
Winter & 2 & 0 & 0 & 0 & 0
\end{tabular}


are not that important here; what matters is the univocal relation between Month and Season (January belongs to Winter only, not to two or more seasons) and this is obtained by taking the support of this matrix, yielding the roll-up matrix

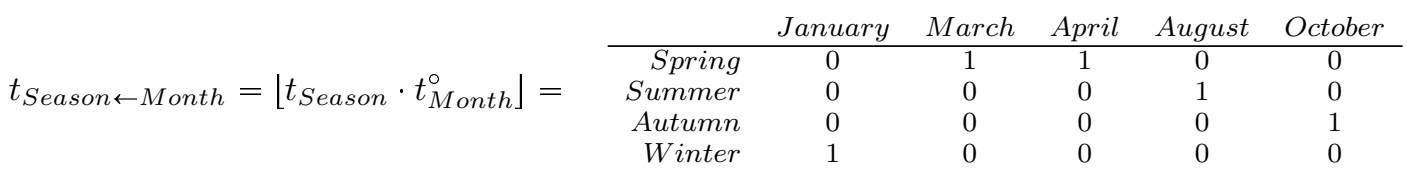

So, given a cross tabulation matrix $|A|<{ }^{X}|C|$, the effect of rolling it up across a given $\mathrm{FD} B \leftarrow A$ is another cross tabulation given by matrix $t_{B \leftarrow A} \cdot X$ of type $|B| \leftarrow|C|$, to which totals can be added, e.g. $\operatorname{tot}\left(t_{B \leftarrow A} \cdot X\right)$. Converse (transpose) caters for the same effect on the right-hand side: rolling $X$ up across another FD $C \leftarrow D$ yields matrix $X \cdot t_{C \leftarrow D}^{\circ}$ of type $|A| \stackrel{X}{\longleftarrow}|D|$. We illustrate this below by instantiating $X$ with a cross tabulation from Model to Month

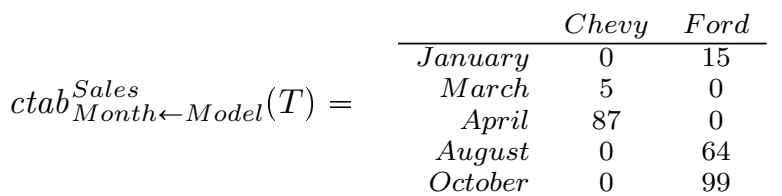

which, once composed with roll-up matrix (36) yields the expected rolling up effect, once equipped with totals:

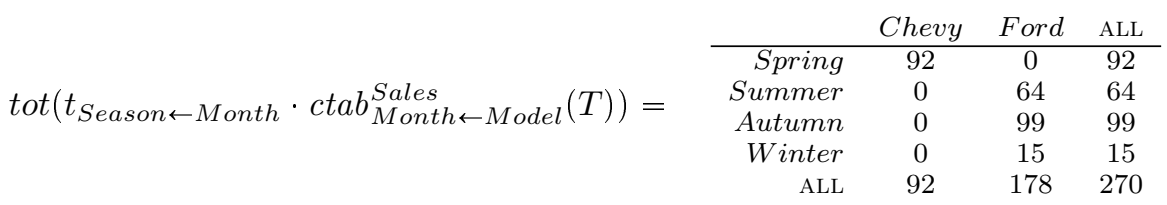

Note that we could have computed $\operatorname{tctab} \underset{\text { Season } \leftarrow \text { Model }}{\text { Sales }}(T)$ in one go, without the help of the roll-up matrix, obtaining the same result as (38). The general result expresses the fusion between roll-up matrices and cross-tabulations as follows:

$$
\operatorname{tot}\left(t_{B \leftarrow A} \cdot \operatorname{ctab}_{A \leftarrow C}^{M}(T)\right)=\operatorname{tctab}_{B \leftarrow C}^{M}(T) \quad \Leftarrow \quad B \leftarrow^{T} A
$$

To prove $\sqrt{39}$ if suffices, looking at the definition of tctab 28), to cancel tot on both sides and prove that $t_{B \leftarrow A} \cdot \operatorname{ctab}_{A \leftarrow C}^{M}(T)=\operatorname{ctab} b_{B \leftarrow C}^{M}(T)$ holds modulo the same side-condition.

Before doing this, let us see a counter-example in which the side condition does not hold: we compose (37) with $t_{\text {Color } \leftarrow \text { Month }}$ (adjacent matrix, on top) obtaining the bottom-left adjacent matrix. This differs from the direct calculation of $c t a b_{\text {Color } \leftarrow \text { Model }}^{\text {Sales }}(T)$ (bottom-right adjacent matrix) because roll-up matrix $t_{\text {Color } \leftarrow \text { Month }}$ does not capture a functional de-

\begin{tabular}{rccccc} 
& January & March & April & August & October \\
\hline Blue & 1 & 0 & 1 & 0 & 1 \\
Green & 0 & 0 & 0 & 1 & 0 \\
Red & 1 & 1 & 0 & 0 & 0
\end{tabular}
pendence: Month does not determine Color, as the January column shows 16

The rest of the proof of $(39)$ relies on properties of matrix supports which are deferred to appendix A

16 The support of matrix 20, given earlier, is another example of roll-up matrix which does not capture a functional dependence: Ford cars can be of any color, for instance. 
Mind that projections are matrices which represent functions:

$$
\begin{aligned}
& t_{B \leftarrow A} \cdot c t a b_{A \leftarrow C}^{M}(T)=c t a b_{B \leftarrow C}^{M}(T) \\
& \Leftrightarrow \quad\{\text { unfold definitions } 35 \text { and } 24 \text { \} } \\
& \left\lfloor t_{B} \cdot t_{A}^{\circ}\right\rfloor \cdot t_{A} \cdot \llbracket T \rrbracket_{M} \cdot t_{C}^{\circ}=t_{B} \cdot \llbracket T \rrbracket_{M} \cdot t_{C}^{\circ} \\
& \Leftarrow \quad\{\text { Leibniz }\} \\
& \left\lfloor t_{B} \cdot t_{A}^{\circ}\right\rfloor \cdot t_{A}=t_{B} \\
& \Leftarrow \quad\{66 \text { in appendix } \mathrm{A}\} \\
& t_{A}^{\circ} \cdot t_{A} \leqslant t_{B}^{\circ} \cdot t_{B} \\
& \Leftrightarrow \quad\{34\} \\
& B<^{T} A
\end{aligned}
$$

Checking for FDs. Construction (35) enables us to check data sets for functional dependencies. In general, FD $B \leftarrow A$ will hold wherever matrix $t_{B} \cdot t_{A}^{\circ}$ is functional, or simple, equivalent to $t_{B \leftarrow A}$ being so. This terminology is imported from relational algebra [BdM97]: a matrix $S$ will be said to be simple iff its image $S \cdot S^{\circ}$ is diagonal ${ }^{17}$ Instantiating $S$ with $t_{\text {Season }} \cdot t_{\text {Month }}^{\circ}$, for instance, it can be checked that its image

\begin{tabular}{rcccc} 
& Spring & Summer & Autumn & Winter \\
\hline Spring & 2 & 0 & 0 & 0 \\
Summer & 0 & 1 & 0 & 0 \\
Autumn & 0 & 0 & 1 & 0 \\
Winter & 0 & 0 & 0 & 4
\end{tabular}

is diagonal, while that of $(20)$

\begin{tabular}{cccc} 
& Blue & Green & Red \\
\hline Blue & 5 & 2 & 3 \\
Green & 2 & 1 & 1 \\
Red & 3 & 1 & 2
\end{tabular}

is not. Thus, FD Color $\leftarrow$ Model does not hold.

\section{Incremental (parallel) construction}

Cross tabulations as defined by formula (28) can be built incrementally under certain conditions. For instance, suppose one is given yesterday's cross tabulation and today's new data. Then today's cross tabulation (in matrix form) will be obtained by adding (matrix-wise) to yesterday's cross tabulation the cross tabulation of today's raw data.

Viewed from another perspective, this property allows one to parallelize the computation of a cross tabulation by partitioning the raw data and then summing up the cross tabulation of each partition of the raw data. Such a property, which can be regarded as generalization of the linearity property that makes linear applications parallel, can be stated by writing, given dimensions $A$ and $B$, measure $M$ and raw data sources $T$ and $T^{\prime}$,

$$
t c t a b_{A \leftarrow B}^{M}\left(T ; T^{\prime}\right)=t c t a b_{A \leftarrow B}^{M} T+t c t a b_{A \leftarrow B}^{M} T^{\prime}
$$

where $T^{\prime \prime}=T ; T^{\prime}$ denotes the append of the two data sources, i.e. $T^{\prime \prime}$ is a raw data table with the records of database $T$ catenated with those of $T^{\prime} . T$ can be regarded as yesterday's raw data and $T^{\prime}$ as the new data, assuming that $T$ has remained the same (no updates, no deletes). Alternatively, one may regard $T ; T^{\prime}$ as a partition of $T^{\prime \prime}$ intended for divide-and-conquer construction of its $t c t a b_{A \leftarrow B}^{M}$ cross tabulation.

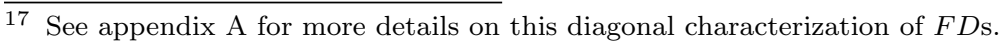


We show below that follows from facts

$$
\begin{aligned}
t_{A}^{\prime \prime} & =\left[t_{A} \mid t_{A}^{\prime}\right] \\
t_{B}^{\prime \prime} & =\left[t_{B} \mid t_{B}^{\prime}\right] \\
\llbracket T ; T^{\prime} \rrbracket_{M} & =\llbracket T \rrbracket_{M} \oplus \llbracket T^{\prime} \rrbracket_{M}
\end{aligned}
$$

where $\oplus$ builds a diagonal matrix by direct sum (11) of two diagonal matrices. Equations (41) and (42) express that projection matrices for $T^{\prime \prime}$ can be built by gluing the corresponding projection matrices $t_{A}, t_{A}^{\prime}$ and $t_{B}, t_{B}^{\prime}$ built for $T$ and for $T^{\prime}$, respectively. Note that, for (41) and (42) to be properly typed, $t_{A}$ and $t_{A}^{\prime}$ (resp. $t_{B}$ and $t_{B}^{\prime}$ ) must have the same target type $|A|$ (resp. $|B|$ ) which can be easily ensured by taking sufficiently large $|A|$ and $|B|$.

To prove facts (41) to (43) we need better definitions for projections (17) and diagonals 22 saving expensive pointwise reasoning. Such definitions and proofs (given in appendix B) can be regarded as a detour needed to smoothly move from first order database notation to linear algebra notation, linking projections (bitmaps) and diagonals to the basic linear algebra of section 4 .

Assuming (41) to (43), the proof of (40) follows from the definition of cross tabulation (28) by a simple equational argument resorting to the laws of matrix algebra:

$$
\begin{aligned}
& \operatorname{tctab}_{A \leftarrow B}^{M}\left(T ; T^{\prime}\right) \\
& =\{28\} \\
& {\left[\frac{t_{A}^{\prime \prime}}{!}\right] \cdot \llbracket T ; T^{\prime} \rrbracket_{M} \cdot\left[\frac{t_{B}^{\prime \prime}}{!}\right]^{\circ}} \\
& =\{41 ; 42 \text { and } 43\} \\
& {\left[\frac{\left[t_{A} \mid t_{A}^{\prime}\right]}{!}\right] \cdot\left(\llbracket T \rrbracket_{M} \oplus \llbracket T^{\prime} \rrbracket_{M}\right) \cdot\left[\frac{\left[t_{B} \mid t_{B}^{\prime}\right]}{!}\right]^{\circ}} \\
& =\{30 \text { twice ; abide law } 10 \text { twice }\} \\
& {\left[\left[\frac{t_{A}}{!}\right] \mid\left[\frac{t_{A}^{\prime}}{!}\right]\right] \cdot\left(\llbracket T \rrbracket_{M} \oplus \llbracket T^{\prime} \rrbracket_{M}\right) \cdot\left[\left[\frac{t_{B}}{!}\right] \mid\left[\frac{t_{B}^{\prime}}{!}\right]\right]^{\circ}} \\
& =\quad\{\text { absorption } 12 \text {; converse-duality } 6 ;\} \\
& {\left[\left[\frac{t_{A}}{!}\right] \cdot \llbracket T \rrbracket_{M} \mid\left[\frac{t_{A}^{\prime}}{!}\right] \cdot \llbracket T^{\prime} \rrbracket_{M}\right] \cdot\left[\frac{\left[\frac{t_{B}}{!}\right]^{\circ}}{\left[\frac{t_{B}^{\prime}}{!}\right]^{\circ}}\right]} \\
& =\quad\{\text { divide and conquer } 77\} \\
& {\left[\frac{t_{A}}{!}\right] \cdot \llbracket T \rrbracket_{M} \cdot\left[\frac{t_{B}}{!}\right]^{\circ}+\left[\frac{t_{A}^{\prime}}{!}\right] \cdot \llbracket T^{\prime} \rrbracket_{M} \cdot\left[\frac{t_{B}^{\prime}}{!}\right]^{\circ}} \\
& =\{28 \text { twice }\} \\
& \operatorname{tctab}_{A \leftarrow B}^{M} T+\operatorname{tctab}_{A \leftarrow B}^{M} T^{\prime}
\end{aligned}
$$

In retrospect, this proof establishes tctab (28) as a structure preserving map (homomorphism) between raw data collection and (cross tabulation) matrix addition, enabling the extraction of parallelism in a formal and direct way. 


\section{Higher-dimensional OLAP}

This section extends cross tabulations towards higher dimensions. The aim is to formulate a basis for a general LA theory for $n$-dimensional OLAP, dealing with all data summary levels presented in $\mathrm{GCB}^{+} 97$, from 0 to 3-dimensional summaries, respectively: aggregate, group-by, cross-tab and cube. The approach goes further by allowing any number $n$ of dimensions.

The proposed generalization depends on the Khatri-Rao product (13) that works as a Cartesian product on matrix types, thus a Cartesian product of the dimensions. As an illustration, remember the projections of our running example and apply the Khatri-Rao product to $t_{\text {Model }}(18)$ and $t_{\text {Color }}(19)$. The outcome is matrix

\begin{tabular}{llllllll} 
& & 1 & 2 & 3 & 4 & 5 & 6 \\
\hline Chevy & Blue & 0 & 1 & 0 & 0 & 0 & 0 \\
Chevy & Green & 0 & 0 & 0 & 0 & 0 & 0 \\
Chevy & Red & 1 & 0 & 0 & 0 & 0 & 0 \\
Ford & Blue & 0 & 0 & 0 & 1 & 0 & 1 \\
Ford & Green & 0 & 0 & 1 & 0 & 0 & 0 \\
Ford & Red & 0 & 0 & 0 & 0 & 1 & 0
\end{tabular}

bearing type $\mid$ Model $\times$ Color $\mid \leftarrow 6$. This tells in which rows the particular dimension pairs appear, compare with Figure 1. Put in other words, this matrix is the higher-rank projection $t_{M o d e l \times C o l o r}$ of the Cartesian product of the two dimensions. In general,

$$
t_{A \times B}=t_{A} \nabla t_{B}
$$

Thus $t_{\text {Model } \times \text { Year } \times \text { Color }}=t_{\text {Model }} \nabla t_{Y \text { ear }} \nabla t_{C o l o r}$, which is projection

\begin{tabular}{lllllllll} 
& & & 1 & 2 & 3 & 4 & 5 & 6 \\
\hline Chevy & 1990 & Blue & 0 & 1 & 0 & 0 & 0 & 0 \\
Chevy & 1990 & Green & 0 & 0 & 0 & 0 & 0 & 0 \\
Chevy & 1990 & Red & 1 & 0 & 0 & 0 & 0 & 0 \\
Chevy & 1991 & Blue & 0 & 0 & 0 & 0 & 0 & 0 \\
Chevy & 1991 & Green & 0 & 0 & 0 & 0 & 0 & 0 \\
Chevy & 1991 & Red & 0 & 0 & 0 & 0 & 0 & 0 \\
Ford & 1990 & Blue & 0 & 0 & 0 & 1 & 0 & 0 \\
Ford & 1990 & Green & 0 & 0 & 1 & 0 & 0 & 0 \\
Ford & 1990 & Red & 0 & 0 & 0 & 0 & 0 & 0 \\
Ford & 1991 & Blue & 0 & 0 & 0 & 0 & 0 & 1 \\
Ford & 1991 & Green & 0 & 0 & 0 & 0 & 0 & 0 \\
Ford & 1991 & Red & 0 & 0 & 0 & 0 & 1 & 0
\end{tabular}

capturing the whole dimensional part of the raw-data table of Figure 1 .

Multidimensional cross tabulations are obtained via the same formula (28) just by supplying higher-rank projections, for instance $t c t a b_{\text {Model } \times \text { Color } \leftarrow \text { Year }}^{\text {Sales }}(T)$ which yields:

\begin{tabular}{llrrr} 
& & 1990 & 1991 & ALL \\
\hline Chevy & Blue & 87 & 0 & 87 \\
Chevy & Green & 0 & 0 & 0 \\
Chevy & Red & 5 & 0 & 5 \\
Ford & Blue & 99 & 7 & 106 \\
Ford & Green & 64 & 0 & 64 \\
Ford & Red & 0 & 8 & 8 \\
ALL & & 255 & 15 & 270
\end{tabular}

corresponding to $A=$ Model $\times$ Color and $B=$ Year in (28). Furthermore, by composing $\llbracket T \rrbracket_{\text {Sales }}$ with the projection of all dimensions given by 45 on the left and totalizing by $!^{\circ}$ on the right, we obtain the following 
column-vector representation of Figure 1

\begin{tabular}{llllr} 
& & & & ALL \\
\cline { 2 - 4 }$t_{\text {Model } \times \text { Year } \times \text { Color }} \cdot \llbracket T \rrbracket_{\text {Sales }} \cdot !^{\circ}=$ & 1990 & Blue & 87 \\
& Chevy & 1990 & Green & 0 \\
& Chevy & 1990 & Red & 5 \\
Chevy & 1991 & Blue & 0 \\
& Chevy & 1991 & Green & 0 \\
Chevy & 1991 & Red & 0 \\
& Ford & 1990 & Blue & 99 \\
& Ford & 1990 & Green & 64 \\
& Ford & 1990 & Red & 0 \\
& Ford & 1991 & Blue & 7 \\
& Ford & 1991 & Green & 0 \\
& Ford & 1991 & Red & 8
\end{tabular}

which, as we shall soon see, is a fragment of the CUBE operator.

A generalization follows from this example. Given a finite, ordered set of dimensions $D$, one calculates the corresponding cube over some given measure attribute by iterating over the powerset $2^{D}$ of $D$, for instance that represented aside for $D=\{M, Y, C\}$ where $M, Y$ and $C$ abbreviate Model, Year and Color, respectively.

Let us denote by $2_{\star}^{D}$ the sequence of all elements of $2^{D}$ ordered in some predefined way induced by the ordering on the dimensions (e.g. $M<Y<C$ ). Thus $2_{\star}^{D}$ is a sequence of (dimension) sequences and we can build the following projection matrix as an iteration of (44) via 32

$$
\begin{aligned}
& t_{2_{\star}^{D}}:\left|2_{\star}^{D}\right| \leftarrow n \\
& t_{2_{\star}^{D}}=[-] \underset{s \leftarrow 2_{\star}^{D}}{\left[\underset{d \leftarrow s}{\nabla} t_{d}\right)}
\end{aligned}
$$

where $[-]$ denotes the finitary extension of vertical blocking (recall section (4) thus stacking up the intermediate projection matrices provided by the innermost iteration.

Note that (47) is not a function (functional matrix) although each contribution $\nabla_{d \leftarrow s} t_{d}$ is so ${ }^{18}$ This redundancy is intentional, as 47 is intended to record all possible combinations of dimension attributes - the shape of the cube. To fill such a shape with the cube contents we multiply by the measure diagonal and totalize with bang converse:

$$
\begin{aligned}
& \operatorname{cube}_{D}^{M}(T):\left|2_{\star}^{D}\right| \leftarrow 1 \\
& \text { cube }_{D}^{M}(T)=t_{2_{\star}^{D}} \cdot \llbracket T \rrbracket_{M} \cdot !^{\circ}
\end{aligned}
$$

Thus the LA representation of a cube is a (column) vector. Aside we show a tabular representation of $c u b e_{\{M o d e l, Y \text { Sear,Color }\}}^{\text {Sales }}(T)$ for our running example. Note the usual convention of filling with ALL marks the "missing attributes" in each $s$ in $2_{\star}^{D}$.

Report MO11a gives a MATLAB script which implements (48). A generic formula for calculating other aggregations on given sub-sequences $S$ of $2_{\star}^{D}$ and measure $M$ from a database table $T$ is given by

$$
\begin{aligned}
& \operatorname{agg}_{S}^{M}(T):|S| \leftarrow 1 \\
& \operatorname{agg}_{S}^{M}(T)=t_{S} \cdot \llbracket T \rrbracket_{M} \cdot !^{\circ}
\end{aligned}
$$

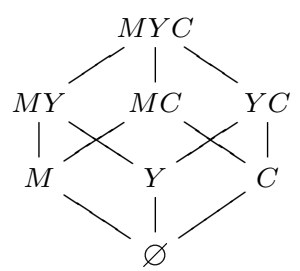

\begin{tabular}{lllr} 
& & & ALL \\
\hline Chevy & 1990 & Blue & 87 \\
Chevy & 1990 & Red & 5 \\
Ford & 1990 & Blue & 99 \\
Ford & 1990 & Green & 64 \\
Ford & 1991 & Blue & 7 \\
Ford & 1991 & Red & 8 \\
Chevy & 1990 & ALL & 92 \\
Ford & 1990 & ALL & 163 \\
Ford & 1991 & ALL & 15 \\
Chevy & ALL & Blue & 87 \\
Chevy & ALL & Red & 5 \\
Ford & ALL & Blue & 106 \\
Ford & ALL & Green & 64 \\
Ford & ALL & Red & 8 \\
ALL & 1990 & Blue & 186 \\
ALL & 1990 & Green & 64 \\
ALL & 1990 & Red & 5 \\
ALL & 1991 & Blue & 7 \\
ALL & 1991 & Red & 8 \\
Chevy & ALL & ALL & 92 \\
Ford & ALL & ALL & 178 \\
ALL & 1990 & ALL & 255 \\
ALL & 1991 & ALL & 15 \\
ALL & ALL & Blue & 193 \\
ALL & ALL & Green & 64 \\
ALL & ALL & Red & 13 \\
ALL & ALL & ALL & 270
\end{tabular}

18 Given two functions $f$ and $g,\left[\frac{f}{g}\right]$ is never a function - it is a relation. Also note that $\left|2_{\star}^{D}\right|=\Sigma_{s \leftarrow 2_{\star}^{D}}|s|$. 
where $t_{S}$ generalizes (47). $S$ tells which dimensions in $D$ are handled and in what order, thus yielding different standard operations for different $S$. For instance, for $S$ containing only the empty sequence $\square$ one has $t_{[]}=$! in 449 , thus obtaining an AGGREGATE $\mathrm{GCB}^{+} 97$ - the grand total block of tot (26 27). At the other extreme, for $S=2_{\star}^{D} \sqrt{49}$ is of course the same as $(48)$, the whole data CUBE. Somewhere between these limit cases one finds, for $S=[s]$ some singleton subsequence of $2_{\star}^{D}$, the GROUP-BY $s$ aggregation. Finally, for $S$ a prefix-closed subsequence of $2_{\star}^{D}-$ for instance, [[Model, Color], [Model], $\left.\square\right]-(49)$ evaluates a ROLL-UP.

The authors of $\mathrm{GCB}^{+97}$ regard GROUP-BY as "an unusual relational operator". While the operator may look "unusual" in the context of the relation algebra which supports the semantics of relational databases, it makes perfect sense in the linear algebra semantics proposed in the current paper for such constructions. Moreover, note that our LA semantics for GROUP-BY not only covers the standard, one-attribute-only case - captured e.g. the SQL syntax aside, which evaluates to

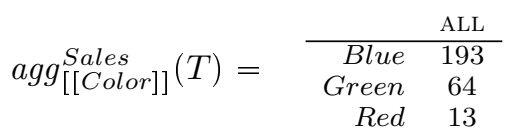

- but also any sequence of grouping attributes — recall e.g. 46, which is the outcome of $a g g_{[[\text {Model,Year,Color }]]}^{\text {Sales }}(T)$. Clearly, any GROUP-BY, AGGREGATE or ROLL-UP is always a fragment of the cube which represents the whole multi-dimensional analysis of the source data.

\section{Related Work}

An overview of data warehousing and OLAP technology can be found in CD97. Since Gray et al delivered their seminal data cube paper in 1996 GBLP96, most work in the field has been concerned with techniques for efficient OLAP, given the small time window (usually at night) when warehouses can go offline for data refreshing.

Another evolution since 1996 is the development of industry standards and specifications. Query languages such as MDX WZP02] relying on multidimensional expressions have emerged as SQL extensions providing the features needed to perform OLAP queries. Our work can be seen as the beginning of a "SQL-free" alternative to provide the same features. We focus on defining a semantics for such features which expresses their meaning in terms of linear algebra operations, ultimately using such meaning to calculate the results.

Yang et al YJA03 focus on the problem of data cube construction and show how a cluster middleware, called ADR (originally developed for scientific data intensive applications) can be used for carrying out scalable implementations of the construction of data cubes.

Bearing the ideal of making OLAP "truly online", $\mathrm{Ng}$ et al NWY01 develop a collection of parallel algorithms directed towards online and offline creation of data cubes using low cost PC clusters to parallelize computations.

Goil and Choudhary GC01 address scalability in multidimensional systems for OLAP and multidimensional analysis and describe the PARSIMONY system providing a parallel and scalable infrastructure for multidimensional online analytical processing, used for both OLAP and data mining. Parallel algorithms are developed for data mining on the multidimensional cube structure for attribute-oriented association rules and decision-tree-based classification.

Literature on "end-to-end" system proposals for parallel OLAP servers is scarce. SIDERA EDD ${ }^{+} 10$ is one such proposal, providing OLAP-specific functionality gathering recent results in a common framework: "the most comprehensive OLAP platform described in the current research literature" [EDD ${ }^{+} 10$ ].

Closer to our approach, Sun and others STF06, STP ${ }^{+} 08$ introduce a technique based on the use of tensors in the area of pattern discovery. (Tensors generalize vectors and matrices, as happens in the mathematical domain, and can be used to represent data-cubes.) To capture temporal evolution one uses tensor streams or sequences that are time indexed structures of tensors, the advantage being a generalization of traditional streams and sequences. On the background stays singular value decomposition (SVD), whose matricial expression conspicuously resembles our starting point (3) and suggests a link between the two approaches which we intend to study in the future. 
Our work also intersects with the area of index-based database-query (response time) optimization, namely in what respects bitmap indices WOS06. Clearly, the projection matrices built in the current paper are bitmaps regarded as matrices. Bitmaps were first implemented in IBM's Model 204 O'N89, becoming a "de facto" device after compression techniques solved their outrageous memory space demands. They are still in use in today's commercial database systems, see WOS06 for details.

\section{Conclusions and Future Work}

This paper addresses the foundations of quantitative data science DP12 from a linear algebra perspective. In particular, it shows how aggregation operations such as cross tabulations and data cubes used in quantitative data analysis can be expressed solely in terms of matrix multiplication, transposition and the Khatri-Rao product. The approach offers potential for deriving a truly algebraic theory of data consolidation, handling the quantitative as well as qualitative sides of data science in an elegant and typed way. Moreover, all operations involved, namely

- the conversion of dimension attributes into projection matrices

- the conversion of measure attributes into diagonal matrices

- the calculation of cross tabulations, and

- the calculation of data cubes

become parallel ("for free") as immediate consequence of the very basic law of divide and conquer (7).

Our main aim is to set up a framework allowing for algebraic reasoning about data analysis operations that have hitherto been described informally or by program code only. The approach is generic and extensible, as much as the underlying mathematics is so. Take for instance the following matrix capturing the Season $\leftarrow$ Month relationship in a more refined way:

\begin{tabular}{|c|c|c|c|c|c|c|c|c|c|c|c|c|}
\hline & 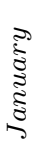 & 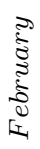 & 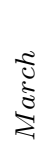 & $\begin{array}{l}\widetilde{\tilde{\Sigma}} \\
\frac{\bar{\sigma}}{\bar{\sigma}}\end{array}$ & $\frac{\overrightarrow{8}}{2}$ & 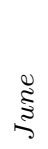 & $\frac{\vec{J}}{\stackrel{\Xi}{J}}$ & 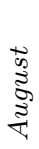 & $\begin{array}{c}\hat{d} \\
\text { है } \\
\text { है } \\
\stackrel{\mathbb{s}}{d} \\
\text { कू }\end{array}$ & 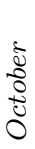 & 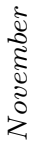 & 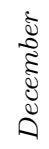 \\
\hline Spring & 0 & 0 & 0.3 & 1 & 1 & 0.7 & 0 & 0 & 0 & 0 & 0 & 0 \\
\hline Summer & 0 & 0 & 0 & 0 & 0 & 0.3 & 1 & 1 & 0.7 & 0 & 0 & 0 \\
\hline Autumn & 0 & 0 & 0 & 0 & 0 & 0 & 0 & 0 & 0.3 & 1 & 1 & 0.7 \\
\hline Winter & 1 & 1 & 0.7 & 0 & 0 & 0 & 0 & 0 & 0 & 0 & 0 & 0.3 \\
\hline
\end{tabular}

In this case, FD Season $\leftarrow$ Month does not strictly hold, for equinoctial and solsticial months are doubly classified in the seasons they border, in different proportions $(70 \%$ for the season which ends, $30 \%$ for the one which starts).

One may say that a "fuzzy" data dependency holds in (50). In spite of the possible complexity that this extension to the standard situation might raise from a traditional OLAP perspective, in our setting it doesn't change anything, as such a "fuzzy" months-into-seasons roll-up process would work precisely in the same way: using this matrix ${ }^{19}$ in $(38)$, for instance, one would obtain

\begin{tabular}{rccc} 
& Chevy & Ford & ALL \\
\hline Spring & 88.5 & 0 & 88.5 \\
Summer & 0 & 64 & 64 \\
Autumn & 0 & 99 & 99 \\
Winter & 3.5 & 15 & 18.5 \\
ALL & 92 & 178 & 270
\end{tabular}

indicating that some (between 3 and 4) of the 92 Chevys sold are likely to have been Winter sales rather than Spring sales. Note that (50) can be regarded as a probabilistic function, meaning that the linear algebra semantics of such functions as studied in e.g. Oli12 can also be useful in this data (rather than algorithmic) context.

19 Pre-composed with the obvious $5 \rightarrow 12$ type coercion matrix embedding five into twelve months, of course. 
Future work. Further research in the direction of thoroughly justifying our approach is under way MO14. In the current paper, the data cube construction is derived from that of cross tabulation. MO14 exploits the alternative view of regarding the data cube as the primitive construction wherefrom the other $2 \mathrm{D}, 1 \mathrm{D}$ and OD aggregators are derived. This makes it easier to prove a number of results, for instance the commutation between cube construction and generic vectorization $\mathrm{MO} 13$.

Moreover, we have to better cross-check our matrix encoding of OLAP (and FDs) with already existing OLAP formal models DT99, PKL02. Mimicking OLAP algebra (whatever this means) in terms of linear algebra may provide better and simpler proofs for existing results and generate new ones, as our experience in pointfree calculation already shows in the relational algebra field Oli14a. This research agenda should also include, of course, a closer look at STF06.

Extending the LA encoding to other forms of data consolidation such as e.g. averaging is within reach. Averaging rather than summing up measure vectors is obtained once again via bang matrices and scalar division, avg $v=(! \cdot v) /\left(! \cdot !^{\circ}\right)$, for $n \longleftarrow^{v} 1$ and $1 \stackrel{!}{\longleftarrow} n$, where $! \cdot v$ reduces vector $v$ to the scalar which records the sum of its elements. Averaging holds since $\left(! \cdot !^{\circ}\right)$ is $1 \leftarrow^{n} 1$, also a scalar. It is easy to see that obtaining cross tabulations consolidated by averaging is a question of augmenting equation (3) with the (index-wise) division of the cross tabulation matrix by the corresponding counting matrix:

$$
\frac{t_{A} \cdot \llbracket T \rrbracket_{M} \cdot t_{B}^{\circ}}{t_{A} \cdot t_{B}^{\circ}}
$$

Extremes (min and max) are achievable by tuning multiplication and sum of matrix elements to suitable semirings. But calculating more exotic data consolidation forms as e.g. population's standard deviation is challenging due to the complexity of the formulas. This is achievable with intensive use of Khatri-Rao products and other non-trivial matrix operations, but further research is needed to evaluate the practicality of such usage.

Another direction for future work is to benchmark a realistic implementation of our approach (derivable from the MATLAB scripts) against existing OLAP systems (e.g. those mentioned in section 9) thus testing whether the parallelism inherent in the LA scripts materializes in real-life applications. Recall that our approach is column-driven. Given that column-store databases for OLAP are being used as an alternative to ROLAP (relational row-driven OLAP) or MOLAP (multidimensional OLAP), it would be interesting to analyze if our LA semantics for OLAP could also improve its processing [Sor12].

Clearly, one needs to be able to process sparse matrices (which our projection bitmaps and diagonals are) as efficiently as possible. Bell and Garland [BG09] explore the design of efficient sparse matrix-vector kernels for throughput oriented processors and implement these kernels in a parallel computing architecture developed by NVIDIA. The OSKI Library $\left[\mathrm{WOV}^{+} 09\right]$ is a collection of low-level $\mathrm{C}$ primitives that provide automatically tuned computational kernels on sparse matrices, for use in solver libraries and applications. OSKI has a BLAS-style interface, providing basic kernels like sparse matrix-vector multiply and sparse triangular solve, among others.

Last but not least, Yang et al YPS11 propose architecture-aware optimizations for sparse matrix multiplication on GPUs and study the impact of their efforts on graph mining. This work is another piece of evidence suggesting that future OLAP and data mining should rely on linear algebra.

\section{Acknowledgements}

The research reported in this paper was funded by project LeanBigData: Ultra-Scalable and Ultra-Efficient Integrated and Visual Big Data Analytics (FP7-619606), by ERDF - European Regional Development Fund through the COMPETE Programme (operational programme for competitiveness) and by National Funds through the FCT - Fundação para a Ciência e a Tecnologia (Portuguese Foundation for Science and Technology) within project FCOMP-01-0124-FEDER-010047.

While doing this work Hugo Macedo held FCT grant number SFRH/BD/33235/2007.

The authors would like to thank the insightful comments, suggestions and references provided by the reviewers. Special thanks go to the reviewer who suggested definition $(72)$ and drafted a version of the proof of 43. Comments by Gabriel David and Orlando Belo on an earlier version of this paper are also gratefully acknowledged. 


\section{References}

[BB04] K. Backhouse and R.C. Backhouse. Safety of abstract interpretations for free, via logical relations and Galois connections. Science of Computer Programming, 15(1-2):153-196, 2004.

[BdM97] R. Bird and O. de Moor. Algebra of Programming. Series in Computer Science. Prentice-Hall International, 1997. C.A.R. Hoare, series editor.

[BG09] N. Bell and M. Garland. Implementing sparse matrix-vector multiplication on throughput-oriented processors. In Proceedings of the Conference on High Performance Computing Networking, Storage and Analysis, SC'09, pages 18:1-18:11, New York, NY, USA, 2009. ACM.

[Bir89] R.S. Bird. Lecture notes on constructive functional programming, 1989. In M. Broy, editor, CMCS Int. Summer School directed by F.L. Bauer [et al.], Springer, 1989. NATO Adv. Science Institute (Series F: Comp. and System Sciences Vol. 55 .

[BM06] R.C. Backhouse and D. Michaelis. Exercises in quantifier manipulation. In T. Uustalu, editor, MPC'06, volume 4014 of LNCS, pages 70-81. Springer, 2006.

[CD97] S. Chaudhuri and U. Dayal. An overview of data warehousing and OLAP technology. SIGMOD Rec., 26:65-74, March 1997.

[Cod70] E.F. Codd. A relational model of data for large shared data banks. CACM, 13(6):377-387, June 1970.

[DGM14] J. Desharnais, A. Grinenko, and B. Möller. Relational style laws and constructs of linear algebra. Journal of Logical and Algebraic Methods in Programming, 83(2):154-168, 2014.

[DP12] T.H. Davenport and D.J. Patil. Data scientist: The sexiest job of the 21st century, Oct 2012. Harvard Business Review.

[DT99] A. Datta and H. Thomas. The cube data model: a conceptual model and algebra for on-line analytical processing in data warehouses. Decision Support Systems, 27(3):289-301, 1999.

$\left[\mathrm{EDD}^{+} 10\right]$ T. Eavis, G. Dimitrov, I. Dimitrov, D. Cueva, A. Lopez, and A. Taleb. Parallel OLAP with the Sidera server. Future Generation Computer Systems, 26(2):259-266, 2010.

[Fri02] M.F. Frias. Fork algebras in algebra, logic and computer science, 2002. Logic and Computer Science. World Scientific Publishing Co.

[GBLP96] J. Gray, A. Bosworth, A. Layman, and H. Pirahesh. Data cube: A relational aggregation operator generalizing group-by, cross-tab, and sub-total. In Stanley Y. W. Su, editor, Proceedings of the 12th Int. Conf. on Data Engineering, Feb.26-Mar.1, 1996, New Orleans, Louisiana, pages 152-159. IEEE Computer Society, 1996.

[GC97] S. Goil and A. Choudhary. High performance OLAP and data mining on parallel computers. Data Mining and Knowledge Discovery, 1:391-417, 1997.

[GC01] S. Goil and A. Choudhary. Parsimony: An infrastructure for parallel multidimensional analysis and data mining. Journal of Parallel and Distributed Computing, 61(3):285-321, 2001.

$\left[\mathrm{GCB}^{+}\right.$97] J. Gray, S. Chaudhuri, A. Bosworth, A. Layman, D. Reichart, M. Venkatrao, F. Pellow, and H. Pirahesh. Data cube: A relational aggregation operator generalizing group-by, cross-tab, and sub-totals. Data Mining and Knowledge Discovery, 1(1):29-53, 1997.

[GL97] M. Gyssens and L.V.S. Lakshmanan. A foundation for multi-dimensional databases. In The VLDB Journal, pages 106-115, 1997.

[JLN00] T. Johnson, L.V. Lakshmanan, and R.T. Ng. The 3w model and algebra for unified data mining. In $V L D B$, pages $21-32,2000$

[JPT10] C.S. Jensen, T.B. Pedersen, and C. Thomsen. Multidimensional Databases and Data Warehousing. Synthesis Lectures on Data Management. Morgan \& Claypool Publishers, 2010.

[Mac12] H. Macedo. Matrices as Arrows - Why Categories of Matrices Matter. PhD thesis, University of Minho, October 2012. MAPi PhD programme.

[Mai83] D. Maier. The Theory of Relational Databases. Computer Science Press, 1983.

[MO10] H.D. Macedo and J.N. Oliveira. Matrices As Arrows! A Biproduct Approach to Typed Linear Algebra. In MPC, volume 6120 of $L N C S$, pages 271-287. Springer, 2010.

[MO11a] H.D. Macedo and J.N. Oliveira. Do the two middle letters of "OLAP" stand for linear algebra ("LA")? Technical Report TR-HASLab:4:2011, HASLab, U.Minho \& INESC TEC, July 2011. available from http://wiki.di.uminho. pt/twiki/bin/view/DI/FMHAS/TechnicalReports

[MO11b] H.D. Macedo and J.N. Oliveira. Towards linear algebras of components. In FACS 2010, volume 6921 of LNCS, pages 300-303. Springer, 2011.

[MO13] H.D. Macedo and J.N. Oliveira. Typing linear algebra: A biproduct-oriented approach. Science of Computer Programming, 78(11):2160-2191, 2013.

[MO14] H.D. Macedo and J.N. Oliveira. Typed linear algebra for the data scientist, 2014. In preparation.

[NWY01] R.T. Ng, A. Wagner, and Y. Yin. Iceberg-cube computation with PC clusters. SIGMOD Rec., 30:25-36, May 2001.

[Oli09] J.N. Oliveira. Extended static checking by calculation using the pointfree transform. volume 5520 of $L N C S$, pages 195-251. Springer-Verlag, 2009.

[Oli11] J.N. Oliveira. Pointfree foundations for (generic) lossless decomposition. Technical Report TR-HASLab:3:2011, HASLab, U.Minho \& INESC TEC, 2011. available from http://wiki.di.uminho.pt/twiki/bin/view/DI/FMHAS/ TechnicalReports

[Oli12] J.N Oliveira. Towards a linear algebra of programming. Formal Aspects of Computing, 24(4-6):433-458, 2012.

[Oli13] J.N. Oliveira. Weighted automata as coalgebras in categories of matrices. Int. Journal of Found. of Comp. Science, 24(06):709-728, 2013. 
[Oli14a] J.N. Oliveira. A relation-algebraic approach to the "Hoare logic" of functional dependencies. JLAP, 83(2):249-262, 2014.

[Oli14b] J.N. Oliveira. Relational algebra for "just good enough" hardware. In RAMiCS, volume 8428 of $L N C S$, pages 119-138. Springer Berlin / Heidelberg, 2014.

[O'N89] P. O'Neil. Model 204 architecture and performance. In Dieter Gawlick, Mark Haynie, and Andreas Reuter, editors, High Performance Transaction Systems, volume 359 of Lecture Notes in Computer Science, pages 39-59. Springer Berlin / Heidelberg, 1989.

[PJ01] T.B. Pedersen and C.S. Jensen. Multidimensional database technology. Computer, 34:40-46, December 2001.

[PKL02] C.-S. Park, M.H. Kim, and Y.-J. Lee. Finding an efficient rewriting of OLAP queries using materialized views in data warehouses. Decision Support Systems, 32(4):379-399, 2002.

[RR98] C.R. Rao and M.B. Rao. Matrix algebra and its applications to statistics and econometrics. World Scientific Pub Co Inc, 1998.

[SBL14] L. Sorber, M. Barel, and L. Lathauwer. Tensorlab v2.0: A MATLAB toolbox for tensor computations, January 2014. Available online, URL: http://www.tensorlab.net.

[Sch11] G. Schmidt. Relational Mathematics, volume 132 of Encyclopedia of Mathematics and its Applications. Cambridge U.P., 2011.

[Sor12] S. Sorjonen. OLAP query performance in column-oriented databases, 2012. Columnar Databases Seminar, DCS, University of Helsinki. Available from: https://www.cs.helsinki.fi/en/courses/58312305/2012/s/s/1.

[STF06] J. Sun, D. Tao, and C. Faloutsos. Beyond streams and graphs: dynamic tensor analysis. In KDD'06: Proc. of the 12th ACM SIGKDD Int. Conf. on Knowledge Discovery and Data Mining, pages 374-383. ACM, 2006.

$\left[\mathrm{STP}^{+} 08\right] \quad$ J. Sun, D. Tao, S. Papadimitriou, P.S. Yu, and C. Faloutsos. Incremental tensor analysis: Theory and applications. ACM Transactions on Knowledge Discovery from Data, 2:11:1-11:37, October 2008.

[VS99] P. Vassiliadis and T. Sellis. A survey of logical models for OLAP databases. SIGMOD Rec., 28(4):64-69, December 1999.

[WOS06] K. Wu, E.J. Otoo, and A. Shoshani. Optimizing bitmap indices with efficient compression. ACM Trans. Database Syst., 31:1-38, March 2006.

$\left[\mathrm{WOV}^{+} 09\right]$ S. Williams, L. Oliker, R. Vuduc, J. Shalf, K. Yelick, and J. Demmel. Optimization of sparse matrix-vector multiplication on emerging multicore platforms. Parallel Comput., 35:178-194, March 2009.

[WZP02] M. Whitehorn, R. Zare, and M. Pasumansky. Fast track to MDX. Springer, 2002.

[YJA03] Ge Yang, Ruoming Jin, and Gagan Agrawal. Implementing data cube construction using a cluster middleware: algorithms, implementation experience, and performance evaluation. Future Generation Computer Systems, 19(4):533$550,2003$.

[YPS11] X. Yang, S. Parthasarathy, and P. Sadayappan. Fast sparse matrix-vector multiplication on GPUs: implications for graph mining. Proceedings of the VLDB Endowment, 4:231-242, January 2011.

\section{A. Appendix on counting matrices and function injectivity}

This appendix is concerned with functional dependencies and their relationship with counting matrices, that is, matrices whose cells are natural numbers. Although some results below hold for arbitrary matrices, we shall restrict to counting matrices for economy of presentation. As special case we have the Boolean matrices, so called because they hold either 0 or 1 in their cells, which can be interpreted as the Boolean truth values. Clearly, a counting matrix $B$ is Boolean iff $B \leqslant \top$, where $T$ denotes the everywhere- 1 matrix of its type obtainable by composing "bang" 30 with its converse: $T=!^{\circ} \cdot !$.

Boolean matrices represent binary relations in matricial form. Given Boolean matrices $B$ and $B^{\prime}, B \leqslant B^{\prime}$ expresses inclusion of the binary relations represented by such matrices, that is $\left\langle\forall y, x:: y B x \Rightarrow y B^{\prime} x\right\rangle$ recalling our use of infix notation $y M x$ to express the cell of matrix $M$ addressed by row $y$ and column $x$ 20

Any function $f$ is a special case of the Boolean matrix such that $y f x=1$ if $y=f x$ and $y f x=0$ otherwise. Note the use of symbol $f$ to denote two mathematical objects, the function itself (as in $y=f x$ ) and its matrix representation (as in $y f x=1$ ). This abuse of notation (common in relation algebra) enables the following rules interfacing index-free and index-wise matrix notation, where $f$ and $g$ functional matrices:

$$
\begin{aligned}
y\left(g^{\circ} \cdot M \cdot f\right) x & =(g y) M(f x) \\
y(f \cdot M) x & =\langle\Sigma z: y=f z: z M x\rangle \\
y\left(M \cdot f^{\circ}\right) x & =\langle\Sigma z: x=f z: y M z\rangle
\end{aligned}
$$

These rules are expressed in the style of the Eindhoven quantifier calculus (see e.g. BM06]) and are convenient shorthands for the corresponding instances of matrix composition (5). Rule (51) extends to typed matrix

20 As advocated in Oli13], this notation finds its inspiration in terms such as e.g. $y \leqslant x$ which one is familiar with since school maths. 
algebra a similar rule known from relation algebra BB04. Note how (52) is obtained from (5) by "trading" Boolean cell $y f z$ with the corresponding Boolean formula $y=f z$, as explained in [Oli13]. This can be done with any other Boolean term $y B x$.

Rule (51) is enough to derive the equalities

$$
\begin{aligned}
! \cdot f & =! \\
g^{\circ} \cdot(M \theta N) \cdot f & =\left(g^{\circ} \cdot M \cdot f\right) \theta\left(g^{\circ} \cdot N \cdot f\right)
\end{aligned}
$$

for suitably typed functions $f$ and $g$ and matrix-cell binary operation $\theta$ promoted to a matrix operator (with the usual notation overloading), that is, $y(M \theta N) x=(y M x) \theta(y N x)$. From (54) and $\mathrm{\top}=!^{\circ} \cdot !$ one immediately draws:

$$
\top \cdot f=\top
$$

Supports. Let $n \in \mathbb{N}_{0}$ be a natural number and define its support $\lfloor n\rfloor=$ if $n \geqslant 1$ then 1 else 0 , that is, $\lfloor n\rfloor=n \downarrow 1$ where $m \downarrow n$ denotes the least of $m$ or $n$. Clearly,

$$
x \leqslant\lfloor n\rfloor \Leftrightarrow x \leqslant n \wedge x \leqslant 1
$$

that is, $\lfloor n\rfloor$ is the largest "Boolean number" (0 or 1$)$ at most $n$. Thus $\lfloor 0\rfloor=0,\lfloor 1\rfloor=1$ and, in general $\lfloor n\rfloor=n \Leftrightarrow n \leqslant 1$ : the support of a "Boolean number" (0 or 1$)$ is itself.

Let us now extend $\lfloor n\rfloor$ from naturals to matrices of naturals (counting matrices): 0 becomes $\perp$, the everywhere-0 matrix of its type; 1 becomes $T$, the everywhere-1 matrix of its type and (57) becomes

$$
X \leqslant\lfloor N\rfloor \Leftrightarrow X \leqslant N \wedge X \leqslant \top
$$

equivalent to the following, closed definition

$$
\lfloor M\rfloor=M \downarrow \top
$$

where $y(M \downarrow N) x=(y M x) \downarrow(y N x)$, overloading $m \downarrow n$.

Cancellation in $(58)$ yields $|N| \leqslant N$ and $|N| \leqslant T$, the latter saying that $|N|$ is a Boolean matrix. All equalities above extend to counting matrices, e.g. $\lfloor N\rfloor=N \Leftrightarrow N \leqslant T$ : the support of a Boolean matrix is itself. Moreover, [-] is a monotonic function from counting to Boolean matrices. From (58) one also obtains (via converses):

$$
\left\lfloor M^{\circ}\right\rfloor=\lfloor M\rfloor^{\circ}
$$

In general $\lfloor M \cdot N\rfloor \neq\lfloor M\rfloor \cdot\lfloor N\rfloor$, since composition is not closed over Boolean matrices $(T \cdot T>T$, for instance). Nevertheless, the special case

$$
\lfloor M \cdot f\rfloor=\lfloor M\rfloor \cdot\lfloor f\rfloor=\lfloor M\rfloor \cdot f
$$

holds:

$$
\begin{aligned}
& \lfloor M \cdot f\rfloor \\
= & \quad\{59 ; ; 56\}\} \\
= & \quad\{(M \cdot f) \downarrow(\top \cdot f) \\
& (M \downarrow \top) \cdot f \\
= & \quad\{59\} \\
& \lfloor M\rfloor \cdot f
\end{aligned}
$$

From (61) the more general rule

$$
\left\lfloor g^{\circ} \cdot M \cdot f\right\rfloor=g^{\circ} \cdot\lfloor M\rfloor \cdot f
$$


can be derived by taking converses:

$$
\begin{aligned}
& \left\lfloor g^{\circ} \cdot M \cdot f\right\rfloor \\
= & \quad \text { contravariance ; idempotence }\} \\
= & \left\lfloor\left(M^{\circ} \cdot g\right)^{\circ} \cdot f\right\rfloor \\
& \quad\{61 ; ; 60\}\} \\
= & \quad\left\{M^{\circ} \cdot g\right\rfloor^{\circ} \cdot f \\
& \left(\lfloor M\rfloor^{\circ} \cdot g\right)^{\circ} \cdot f \\
= & \quad\{\text { contravariance }\} \\
& g^{\circ} \cdot\lfloor M\rfloor \cdot f
\end{aligned}
$$

A counting matrix $M$ is diagonal iff $|M| \leqslant i d$, that is, $\langle\forall y, x: y \neq x: y M x=0\rangle$. An example of diagonal matrix is the image $g \cdot g^{\circ}$ of a function $g$ since, by $(53), b^{\prime}\left(g \cdot g^{\circ}\right) b=\left\langle\Sigma a: b=g a: b^{\prime} g a\right\rangle$ which is the same as $\left\langle\Sigma a: b^{\prime}=g a \wedge b=g a: 1\right\rangle$ trading term $b^{\prime}=g a$, since $g$ is a function. Thus

$$
b^{\prime}\left(g \cdot g^{\circ}\right) b=\left\langle\sum a: b^{\prime}=g a \wedge b=g a \wedge b^{\prime}=b: 1\right\rangle
$$

and therefore $b^{\prime}\left(g \cdot g^{\circ}\right) b=0$ for $b^{\prime} \neq b$.

Functional injectivity. For $M:=i d$ one draws from $(62)$ that $g^{\circ} \cdot f$ is Boolean, $\left\lfloor g^{\circ} \cdot f\right\rfloor=g^{\circ} \cdot f$. Thus the kernel of a function $f$ Oli14a

$$
f^{\circ} \cdot f=\left\lfloor f^{\circ} \cdot f\right\rfloor
$$

is Boolean. By (51), Leibniz rule $x^{\prime}=x \Rightarrow f x^{\prime}=f x$ encodes into $i d \leqslant f^{\circ} \cdot f$, whereby one obtains (by monotonicity) $g \leqslant g \cdot f^{\circ} \cdot f$ and

$$
g \leqslant\left\lfloor g \cdot f^{\circ}\right\rfloor \cdot f
$$

by taking supports and $(61)$.

Functions can be compared by comparing their kernels: by unfolding $f^{\circ} \cdot f \leqslant g^{\circ} \cdot g$ once again by (51), we get:

$$
\begin{aligned}
& x^{\prime}\left(f^{\circ} \cdot f\right) x \leqslant x^{\prime}\left(g^{\circ} \cdot g\right) x \\
\Leftrightarrow & \quad\{\quad \text { 51 twice }\} \\
& \left(f x^{\prime}\right) i d(f x) \leqslant\left(g x^{\prime}\right) i d(g x) \\
\Leftrightarrow \quad & \quad\{b(i d) a \text { encodes } b=a \text { and } \leqslant \text { over }\{0,1\} \text { encodes implication }\} \\
& f x^{\prime}=f x \Rightarrow g x^{\prime}=g x
\end{aligned}
$$

Colloquially: " $g$ does not distinguish what $f$ regards as equal". Formally: $g$ is less injective than $f{ }^{21}$ The following result

$$
\left\lfloor g \cdot f^{\circ}\right\rfloor \cdot f=g \Leftarrow g \text { is less injective than } f
$$

$\overline{21}$ Cf. e.g. Oli14a, where the same inequality is handled relationally. 
is required in section 6 of the current paper and relies on the injectivity ordering on functions:

$$
\begin{aligned}
& \left\lfloor g \cdot f^{\circ}\right\rfloor \cdot f=g \\
& \Leftrightarrow \quad\{65\} \\
& \left\lfloor g \cdot f^{\circ}\right\rfloor \cdot f \leqslant g \\
& \Leftarrow \quad\left\{\left\lfloor g \cdot g^{\circ}\right\rfloor \cdot g \leqslant g \text { by monotonicity of composition since } g \cdot g^{\circ} \text { is diagonal } 63\right\} \\
& \left\lfloor g \cdot f^{\circ}\right\rfloor \cdot f \leqslant\left\lfloor g \cdot g^{\circ}\right\rfloor \cdot g \\
& \Leftrightarrow \quad\{61 \text { twice }\} \\
& \left\lfloor g \cdot f^{\circ} \cdot f\right\rfloor \leqslant\left\lfloor g \cdot g^{\circ} \cdot g\right\rfloor \\
& \Leftarrow \quad\{\text { monotonicity of [-] and of composition } \\
& f^{\circ} \cdot f \leqslant g^{\circ} \cdot g
\end{aligned}
$$

\section{B. Appendix on bitmaps, projections and diagonals}

Bitmaps. Suppose an array $a=\left[\begin{array}{llll}d_{1} & d_{2} & \ldots & d_{n}\end{array}\right]$ holds $n$ elements of data type $D$. This uniquely determines the function $f_{a}: n \rightarrow D$ such that $f_{a}(i)=d_{i}$ for $1 \leqslant i \leqslant n$, that is, $f_{a}$ tells which datum lives in which position of array $a$. Once such a function is represented as a Boolean matrix of type $D \leftarrow n$ one obtains a bitmap matrix representation of $a$. Note that $a$ itself can be regarded as a generalized $\left.\right|^{22} D$-valued row vector of type $1 \leftarrow n$. Let this change of representation can be captured by function

$$
b m:\left(1_{D} \leftarrow n\right) \rightarrow(D \leftarrow n)
$$

where notation $1_{D} \leftarrow n$ is intended to warn the reader that cells in $b m$ 's input are of type $D$, possibly not a semiring essential for matrix composition to work. (More about this below.) We define $b m$ inductively as follows: for $n=1, b m d_{1}=D<\underline{d_{1}}$, the Boolean (column) vector representing constant function $d_{1}$; for $n>1,-\mathrm{bm}$ - is defined by:

$$
b m\left[a_{1} \mid a_{2}\right]=\left[b m a_{1} \mid b m a_{2}\right]
$$

Let a given raw data table $T$ have $n$ rows (records) and as many columns as the set of its attributes $S=\{A, B, \ldots\}$. Then $T$ may also be regarded as a generalized matrix of type $n \leftarrow S$ whereby the raw-data append operation $T ; T^{\prime}$ (catenation of $T$ with $T^{\prime}$ ) is faithfully captured in matrix block notation by

$$
T ; T^{\prime}=\left[\frac{T}{T^{\prime}}\right]
$$

since both $T$ and $T^{\prime}$ share the same input type $S$. For $A \in S$, constant function $S \ll \underline{A}$ is a Boolean vector with 0 s everywhere but a 1 in the row addressed by attribute $A \in S$. Then $T(A, n)$, the value of attribute $A$ in the $n$-th row of $T$ can be re-written as follows:

$$
\begin{aligned}
& T(A, n) \\
= & \quad \quad \quad \text { using infix notation once } T \text { is regarded as a } n \leftarrow S \text { matrix }\} \\
& n T A \\
= & \quad \quad \quad \text { since } S \leftarrow \underline{A}-1 \text { is a constant function }\} \\
& n T(\underline{A} 1)
\end{aligned}
$$

\footnotetext{
$\overline{22}$ Generalized in the sense that it will hold any kind of heterogeneously typed data, not just numerical data.
} 


$$
\left.\begin{array}{rl}
n(T \cdot \underline{A}) 1 & \{51
\end{array}\right\}
$$

Thus

$$
n \stackrel{T \cdot \underline{A}}{\longleftarrow} 1
$$

is the (column) vector which represents the $A$-column of $T$. This can be turned into a bitmap via $b m$,

$$
t_{A}=b m(T \cdot \underline{A})^{\circ}
$$

providing a pointfree alternative to (17), as well as

$$
t_{A}^{\prime}=b m\left(T^{\prime} \cdot \underline{A}\right)^{\circ}
$$

for another raw-data set $T^{\prime}$ sharing $A$-values in the same range type $|A|$.

Fact (41) can then be calculated as follows:

$$
\begin{aligned}
& {\left[t_{A} \mid t_{A}^{\prime}\right] } \\
= & \quad\{[70 \text { twice }\} \\
= & \quad\left\{b m(T \cdot \underline{A})^{\circ} \mid b m\left(T^{\prime} \cdot \underline{A}\right)^{\circ}\right] \\
& b m\left[(T \cdot \underline{A})^{\circ} \mid\left(T^{\prime} \cdot \underline{A}\right)^{\circ}\right] \\
= & \quad \quad \quad \text { converse-duality }[6\} \\
& b m\left[\frac{T \cdot \underline{A}}{T^{\prime} \cdot \underline{A}}\right]^{\circ} \\
= & \quad\{\text { fusion }[9\} \\
& b m\left(\left[\frac{T}{T^{\prime}}\right] \cdot \underline{A^{\circ}}\right) \\
= & \left.\quad\left\{\text { define } T^{\prime \prime}=\left[\frac{T}{T^{\prime}}\right]=T ; T^{\prime} 68\right\}\right\} \\
& b m\left(T^{\prime \prime} \cdot \underline{A^{\circ}}\right) \\
= & \quad\{70\}\} \\
& t_{A}^{\prime \prime} \quad
\end{aligned}
$$

The proof of 42 is the same, for attribute $B$ instead of $A$.

Diagonals. Back to $(22)$, let $M \in S$ be a measure attribute and let us rely on $(69)$ to capture its diagonalization, via 14:

$$
\llbracket T \rrbracket_{M}=(T \cdot \underline{M})^{\circ} \nabla i d
$$


This definition is convenient for proving fact (43), as follows:

$$
\begin{aligned}
& \llbracket T ; T^{\prime} \rrbracket_{M} \\
= & \{\llbracket 68\} \\
& \llbracket\left[\frac{T}{T^{\prime}}\right] \rrbracket_{M} \\
= & \quad\{\text { definition }\{72\} \\
& \left(\left[\frac{T}{T^{\prime}}\right] \cdot \underline{M}\right)^{\circ} \nabla i d \\
= & \quad\{\text { fusion }\{9 ; ; i d \oplus i d=i d\} \\
= & {\left[\frac{T \cdot \underline{M}}{T^{\prime} \cdot \underline{M}}\right]^{\circ} \nabla(i d \oplus i d) } \\
& \quad\left\{(T \cdot \underline{M})^{\circ} \mid\left(T^{\prime} \cdot \underline{M}\right)^{\circ}\right] \nabla(i d \oplus i d) \\
= & \quad\left\{\left[16 \text { since } T \cdot \underline{M} \text { and } T^{\prime} \cdot \underline{M} \text { are row vectors }\right\}\right. \\
& \left((T \cdot \underline{M})^{\circ} \nabla i d\right) \oplus\left(\left(T^{\prime} \cdot \underline{M}\right)^{\circ} \nabla i d\right) \\
= & \quad\{\text { definition } \underline{72} \text { twice }\} \\
& \llbracket T \rrbracket_{M} \oplus \llbracket T^{\prime} \rrbracket_{M}
\end{aligned}
$$

Recall from section 7 that 443 is central to showing that cross tabulation evaluation is parallelizable 40 . 